7

SECCIÓN: Teorías geográficas, geografía de la cultura y la vida cotidiana
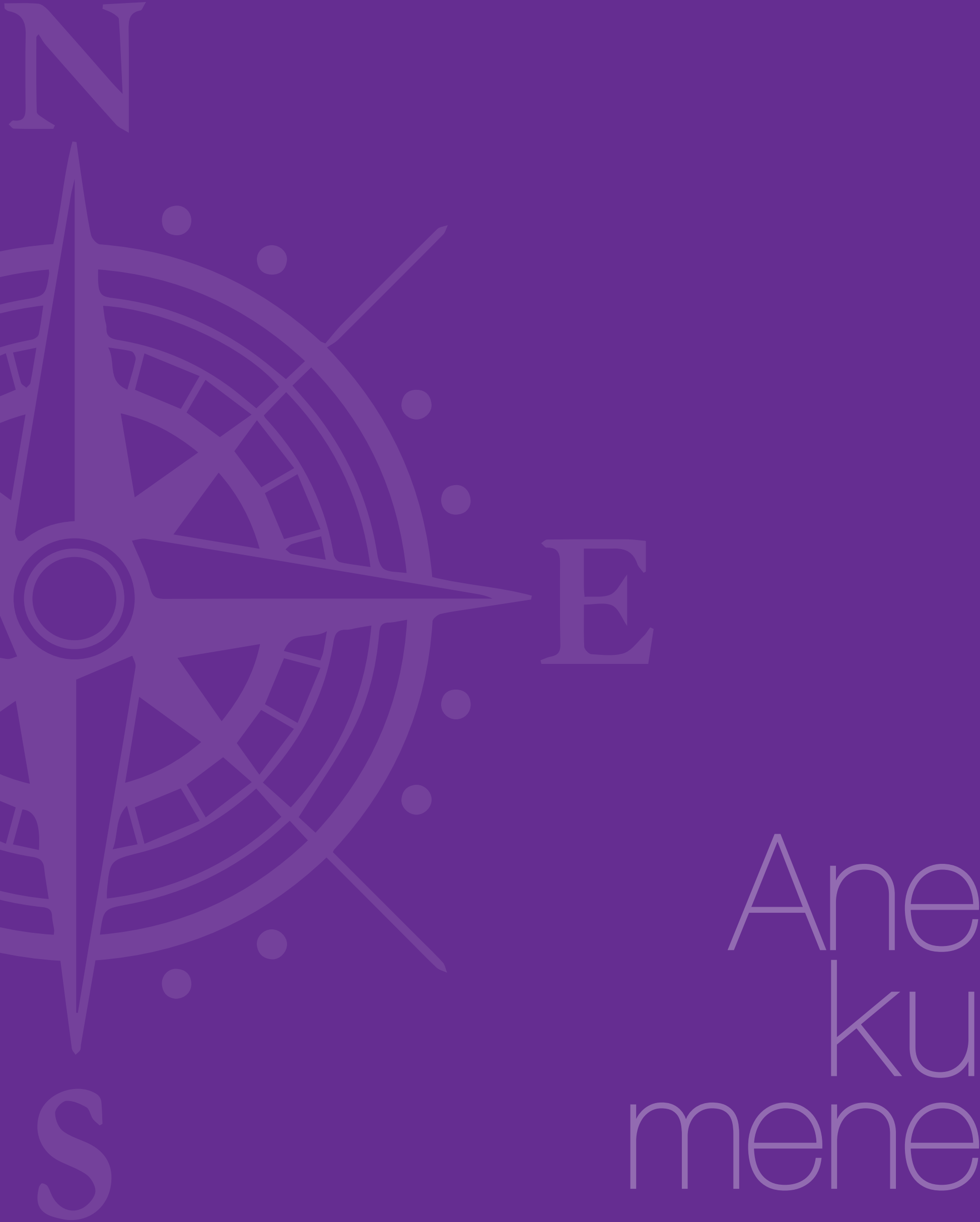


\section{A teoria de Basil Bernstein: caminhos possíveis para a pesquisa em ensino de geografia}

\section{Basil Bernstein's Theory: Possible Paths for Research in the Geography Teaching \\ La teoría de Basil Bernstein: posibles caminos para la investigación sobre enseñanza de la geografía}

Daniel Luiz Stefenon*

Sonia Maria Vanzella Castellar ${ }^{* *}$

\section{Resumo}

A teoria de Basil Bernstein $(1981 ; 1996 ; 1999)$ apresenta elementos importantes para se refletir sobre o problema das desigualdades educacionais reproduzidas no interior da escola, especialmente no âmbito de sociedades marcadas por profundas desigualdades sociais. 0 presente texto destaca de sua produção a teoria dos códigos, os diferentes discursos sujeitos à transformação pedagógica e a teoria do dispositivo pedagógico, a fim de propor diferentes categorias de análise e possíveis caminhos para a pesquisa no Ensino de Geografia. Ao discutir as categorias relações inter/intradisciplinares, discursos verticais e horizontais, discursos instrucionais e regulativos, sequenciamento e compassamento, entre outras possibilidades, este texto apresenta alternativas teóricas e metodológicas para focalizar os discursos curriculares, suas diferentes formas de recontextualização no espaço da escola e o papel desta instituição na democratização dos acessos ao conhecimento e ao mundo de maneira ampla e irrestrita.

\section{Palavras-chave}

Basil Bernstein; desigualdades educacionais; currículo; pesquisa; ensino de geografia

* Professor da Universidade Estadual do Centro Oeste (Unicentro), Campus de Irati, Paraná.

** Profesora Titular de la Universidad de São Paulo, Brasil. 


\begin{abstract}
The theory of Basil Bernstein $(1981,1996,1999)$ presents important elements for reflecting on the problem of educational inequalities in schools, particularly in societies marked by deep social inequalities. This text emphasizes its production about the theory of the codes, the different discourses subject to pedagogical transformation and the pedagogical device theory, in order to propose different categories of analysis and possible paths for the research in the teaching of geography. When discussing the categories of inter/intradisciplinary relations, vertical and horizontal discourses, instructional and regulative discourses, sequencing and pacing, among other possibilities, this text presents theoretical and methodological alternatives to focus on curricular discourses, their different forms of recontextualization in school and the role of this institution in the democratization of access to knowledge and the world in a broad and unrestricted way.
\end{abstract}

\section{Resumen}

La teoría de Basil Bernstein $(1981,1996,1999)$ presenta elementos importantes para reflexionar sobre el problema de las desigualdades educativas que ocurren en las escuelas, especialmente en el ámbito de sociedades marcadas por profundas desigualdades sociales. El presente texto destaca de su producción la teoría de los códigos, los diferentes discursos sujetos a la transformación pedagógica y la teoría del dispositivo pedagógico, con el fin de proponer diferentes categorías de análisis y posibles caminos para la investigación en la enseñanza de la geografía. Al discutir las categorías relaciones inter/intradisciplinarias, discursos verticales y horizontales, discursos instructivos y regulatorios, secuenciación y ritmo, entre otras posibilidades, este texto presenta alternativas teóricas y metodológicas para focalizar los discursos curriculares, sus diferentes formas de recontextualización en el espacio de la escuela y el papel de esta institución en la democratización de los accesos al conocimiento y al mundo de manera amplia e irrestricta.

\section{Keywords}

Basil Bernstein; educational inequalities; curriculum; research geography teaching

\section{Palabras clave}

Basil Bernstein; desigualdades educativas; currículum; investigación; enseñanza de la geografía 
As desigualdades de acesso a diferentes e específicas formas de saberes no âmbito escolar são um ponto central na pauta de desafios que se impõem à educação pública brasileira na contemporaneidade. Parece se consolidar no sistema educacional de nosso país aquilo que Libâneo (2012) classificou como dualidade perversa, onde para os ricos se oferece uma escola de conhecimento, enquanto aos pobres resta uma escola que apenas promove seu acolhimento social. Por estar preocupada com a distribuição das diferentes formas de discurso entre as classes sociais e também com o controle simbólico das consciências exercido pela escola, a teoria de Basil Bernstein (1981; 1996; 1999), a qual pretende-se aqui explorar, parece oferecer um conjunto poderoso de elaborações teóricas e metodológicas para a compreensão do referido problema, inclusive sobre a parte que cabe ao campo da pesquisa em ensino de Geografia.

Este pressuposto foi por nós explorado ao longo da execução de uma pesquisa de doutorado, vinculada ao Programa de Pós-Graduação da Faculdade de Educação da Universidade de São Paulo. O objetivo central da referente pesquisa consistia em compreender as formas pelas quais os saberes geográficos são recontextualizados em diferentes contextos de sala de aula e sua relação com o processo de construção de um currículo promotor de acesso efetivo ao conhecimento escolar, visto aqui como "conhecimento poderoso" (Young, 2007, p. 1294), ou seja, aquele que é capaz de se colocar como um propulsor de mudanças nas consciências e no mundo.

Partindo das elaborações construídas ao longo do desenvolvimento da pesquisa acima citada, o presente texto procura refletir sobre possíveis caminhos para a pesquisa em ensino de Geografia, inaugurados pela perspectiva teórico-metodológica desenvolvida por Bernstein e colaboradores. Para isso, buscou-se incialmente construir uma síntese fundamental da teoria e de seus componentes centrais, para depois apontar aspectos metodológicos dela derivados, destacando inclusive categorias de análise possíveis para as investigações no campo do ensino de Geografia, especialmente as que se interessam sobre sua dimensão curricular.

\section{A teoria de Basil Bernstein}

Bernstein insere-se dentro de um movimento de renovação do pensamento sociológico britânico denominado Nova Sociologia da Educação (NSE), que representou a versão britânica dos movimentos de reação ao funcionalismo das correntes curriculares tradicionais. Tal movimento inaugurou uma perspectiva de pensamento curricular fundamentada numa visão crítica e desconstrutora acerca da relação entre poder e conhecimento nos programas escolares.

De acordo com Marcuschi (1975), da filosofia de Ernst Cassirer e da psicologia de Vigotski e demais autores da escola russa, Bernstein assumiria a ideia de linguagem enquanto um sistema regulador e orientador da consciência. Em Marx e Durkheim, Bernstein encontra as bases para pensar as macroestruturas sociais e as tensões que conformam o mundo, enquanto o interacionismo simbólico presente na psicologia social de George Herbert Mead lhe fornece os referencias para a compreensão das microestruturas psicológicas e as conexões entre sujeito e sociedade. Tudo isto o torna um intelectual de origens plurais, preocupado com a dialética entre sujeito e estrutura na determinação dos mecanismos de linguagem e, por conseguinte, na relação entre os processos de reprodução cultural e de resistência, o que se tornaria, aliás, uma preocupação que o acompanhou ao longo de todo o desenvolvimento de suas produções.

O sistema teórico empreendido por Bernstein é composto por três modelos teóricos principais. O primeiro é o modelo da reprodução e da transformação cultural, que traz uma importante ferramenta para compreender como ocorre a relação e regulação entre diferentes contextos de produção textual, ou seja, como os discursos são produzidos e considerados legítimos dentro de determinados contextos de comunicação (Bernstein, 1981; 1996). Este modelo teórico é também chamado de teoria dos códigos. O código, conceito central do modelo, não é o conteúdo em si da comunicação, mas sim sua gramática profunda, ou seja, um conjunto de regras que dá legitimidade à produção dos textos e representações sociais. Em outras palavras, os códigos correspondem ao "invisível" da comunicação, àquilo que está em seu subterrâneo, enquanto as "práticas interativas" e as "produções textuais" representam a sua dimensão "visível” e concreta (Bernstein, 1996, p. 32).

Ao fazer essa distinção entre o conteúdo da comunicação e as suas regras de realização, Bernstein elabora uma crítica contundente com relação às diferentes perspectivas que explicam o chamado fracasso escolar. Conforme apontado por Dubet (2008), Charlot (2005) e o próprio Bernstein (1996), entre muitos profissionais e intelectuais da educação, é bastante aceita a ideia de que a responsabilidade pelo fracasso escolar, assim como pelo o que se considera sucesso na escola, estaria unicamente nos atributos inerentes à família ou à comunidade da qual se origina o aluno, e teria relação com um certo conjunto de dons naturais que os sujeitos carregam consigo. No entanto, para Bernstein (1996, pp. 166-167), "o sucesso ou o fracasso são uma função do currículo dominante da escola, o qual atua seletivamente sobre aqueles que podem adquiri-lo". Dessa forma, ao invés de assumir um conceito de uma escola passiva perante a realidade, a proposta do autor se configura como uma potente ferramenta de crítica com relação aos seus currículos e práticas pedagógicas. Para ele, currículos e práticas tendem a se constituir como um "texto privilegiante", ou seja, um texto que confere privilégio a determinadas classes sociais, gêneros e identidades culturais na escola, partindo da consideração de que determinadas crianças, de maneira privilegiada, já possuem em casa "uma certa versão" da prática pedagógica que lhe é oferecida na escola, o que lhes dá melhores condições para compreender as regras de comunicação pedagógicas. Em outras palavras, por maior 
que seja a força das estruturas sociais sobre os recursos de comunicação dos sujeitos-estudantes, também é da escola a responsabilidade pelo sucesso ou fracasso de seus alunos.

Propõe ainda uma categorização de diferentes formas de discurso - discursos horizontal (DH) e vertical (DV) (Bernstein 1999) -, a qual busca compreender os princípios internos e a base social de construção dos discursos sujeitos a transformação pedagógica, evidenciando importantes questões acerca das escolhas curriculares e sobre o que realmente conta como conhecimento escolar. O DH, segundo o autor, está diretamente ligado ao senso comum, e por isso caracteriza-se por uma transmissão oral e por uma inscrição local. Isso significa dizer que os significados do DH são diretamente dependentes dos contextos de sua realização. Por sua vez, o DV diz respeito ao conhecimento disciplinar especializado, estruturado com base em padrões de coerência explícitos, sendo hierarquicamente organizado. Os significados do DV independem do contexto de sua evocação, ou seja, dizem respeito a generalizações efetuadas a partir de critérios conhecidos e de formas de realização sistemáticas e universais (Bernstein, 1999; Morais \& Neves, 2007a). De acordo com o autor, a fim de buscar uma maior motivação dos estudantes, especialmente os provenientes de classe social menos privilegiada, flexibiliza-se o currículo escolar, oferecendo a esses estudantes um currículo fortemente fundamentado em DHs, o que limitaria seu acesso ao conhecimento de natureza generalista e abstrata, gerando desigualdades de acesso aos saberes científicos, especialmente.

Por fim, destaca-se a teoria do dispositivo pedagógico, da qual deriva o modelo do discurso pedagógico. Estes apresentam uma descrição acerca dos processos de produção, recontextualização e reprodução dos discursos pedagógicos, e sua relação com os mecanismos de poder e controle de uma dada sociedade (Bernstein, 1996). Os discursos pedagógicos, para Bernstein, são produzidos e reproduzidos a partir de sucessivas etapas de recontextualização. Dentro dessa perspectiva, o conhecimento pedagógico é resultado de um processo de relocação e de refocalização dos discursos disciplinares especializados, os quais são inseridos dentro de uma lógica discursiva normativa chamada por ele de discurso regulativo.

Ao relocar e refocalizar o discurso especializado original, designado por discurso instrucional, a recontextualização o transforma, configurando-o como "outra coisa". Ao retirar o conhecimento disciplinar de seu contexto e recolocá-lo em um outro com natureza e intencionalidades distintas das de sua produção, o processo de recontextualização não realiza a simples redução da complexidade do conhecimento, adaptando-o ao nível de compreensão dos estudantes, mas sim promove sua reinvenção, em consonância com os princípios dominantes que fundam o sistema educativo de uma dada sociedade.

Esse processo de recontextualização ocorre em diferentes instâncias, desde os campos de recontextualização oficial (formulação de currí- culos oficiais), até os campos de recontextualização pedagógicos, seja na produção de livros e materiais didáticos como também pelo próprio professor no processo comunicativo que se desenvolve na sala de aula.

De acordo com Bernstein (1996), ainda com a justificativa de flexibilizar o currículo e torná-lo significativo aos diferentes grupos de uma sociedade, podem ocorrer situações em que para indivíduos de determinadas classes sociais seja apenas oferecido o conhecimento de ordem regulativa, ou seja, de orientação moral e comportamental. Enquanto isso, para grupos de indivíduos mais privilegiados socialmente, os saberes escolares se fundamentam em discursos instrucionais especializados. Esse processo, de acordo com o autor, é um poderoso agente gerador de desigualdades educacionais, e atua como um mecanismo de controle social, limitando determinados grupos ao acesso a determinadas formas de conhecimento elaboradas historicamente pela humanidade.

Ao efetuar sua leitura da teoria de Bernstein, a partir do qual avança propositivamente no debate, Young $(2007$; 2011) argumenta em favor do que chama de conhecimento poderoso. Para o autor, a teoria de Bernstein deixa claro que a escola necessita privilegiar, no enfoque do currículo, uma ideia de saber escolar que "refere-se ao que o conhecimento pode fazer, como, por exemplo, fornecer explicações confiáveis ou novas formas de se pensar a respeito do mundo" (Young, 2007, p. 1294), a fim de buscar mitigar as profundas desigualdades sociais e escolares.

O projeto de escola contido em Bernstein, dessa maneira, é a escola da possibilidade e da transformação; vai além da ideia da exclusividade de acolhimento e de reunião; e se coloca como um locus de questionamento sobre quem se é, sobre o que se sabe e sobre as diferentes escalas de tempo e espaço nas quais se vive.

\section{Configurações metodológicas de pesquisa a partir da teoria de Bernstein}

A perspectiva de investigação sugerida por Bernstein (2000, apud Morais \& Neves, 2007a; 2007b) fundamenta-se em uma relação dialética entre teoria e prática. A teoria é tratada aqui como uma linguagem interna de descrição, enquanto os procedimentos práticos da pesquisa, mais intimamente ligados ao mundo empírico da investigação, são designados como linguagem externa de descrição.

Conforme os desenvolvimentos empreendidos pelas pesquisadoras Ana Maria Morais e Isabel Pestana Neves no âmbito de seu trabalho no grupo de pesquisa ESSA ${ }^{1}$, a linguagem interna de descrição é composta pela própria teoria de Bernstein, sendo que a linguagem externa se refere a

1 O grupo de pesquisas ESSA (Estudos Sociológicos da Sala de Aula), sediado na Universidade de Lisboa (Portugal), é coordenado pelas pesquisadoras Ana Maria Morais e Isabel Pestana Neves, e tem a teoria de Bernstein como uma ferramenta 
um conjunto de categorias e procedimentos de investigação que visam conectar a teoria aos textos e contextos que caracterizam a realidade empírica da pesquisa.

Dessa maneira, ao mesmo tempo em que a teoria busca explicar as relações sociais da atividade pedagógica mediante a construção de uma representação legítima dela, a realidade age reciprocamente sobre ela, produzindo alterações e aperfeiçoamentos no modelo teórico. A linguagem externa de descrição, ou seja, os procedimentos metodológicos, categorias e proposições geradas no processo de investigação, é o que permite essa dialética, sendo um dos elementos mais importantes do trabalho do pesquisador (Morais \& Neves, 2007a; 2007b).

Os procedimentos de pesquisa considerados adequados para o trabaIho de investigação dentro dessa perspectiva são aqueles que se situam entre as abordagens racionalistas e naturalistas de ciência (Morais \& Neves, 2007a; 2007b). Como abordagens racionalistas podem ser designadas aquelas que possuem ênfase sobre o teórico/experimental, de caráter dedutivista. Partem de um pressuposto nomotético, onde teorias são testadas por meio de métodos e metodologias mais objetivos, geralmente de ordem quantitativa, a fim de produzir novas generalizações sobre os objetos. Já a abordagem naturalista é conduzida a partir de pressupostos ideográficos, de caráter indutivista, enfatizando assim aspectos qualitativos e procedimentos de pesquisa fundamentados na etnografia. Esta segunda abordagem tende a se concentrar sobre elementos subjetivos da realidade, que possuem relação direta com o mundo dos significados construídos pelos atores de determinados contextos sociais e educacionais, o qual é o caso da pesquisa que dá origem às reflexões contidas neste texto.

A teoria de Bernstein aponta para a construção de metodologias de investigação que contenham elementos de ambas as abordagens, tratadas aqui como mistas, pois admite a complexidade do objeto em questão, que ao mesmo tempo encerra componentes de ordem objetiva e subjetiva. Isso quer dizer que, trabalhar na pesquisa em educação, dentro da perspectiva bernsteiniana, significa considerar aspectos estruturais e estruturantes, assim como o universo amplo de significações subjetivas que caracterizam os grupos sociais, incluindo-se aí suas perspectivas de futuro, desejos, conflitos e visões de mundo.

Dentro dessa perspectiva de investigação, procedimentos quantitativos e qualitativos não são vistos como antagônicos, mas complementares, sendo que tanto os questionários fechados como entrevistas e práticas de observação, por exemplo, podem se complementar no processo de compreensão do fenômeno em estudo. Por sua vez, o tratamento dos dados dentro do modelo é feito tanto por procedimentos estatísticos

teórico-metodológica central. O ESSA desenvolve diferentes pesquisas dentro do campo da sociologia da educação, produzindo relevantes contribuições para o ensino de ciências e áreas afim (ESSA, 2015). como também por análise interpretativa de conteúdo, expressando assim esse caráter misto da metodologia, a qual busca legitimar suas considerações a partir da triangulação de dados de diferentes origens e significações. Concorda-se aqui com a ideia de que

a triangulação significa a combinação entre diversos métodos qualitativos, mas também a combinação entre métodos qualitativos e quantitativos. Neste caso, as diferentes perspectivas metodológicas complementam-se para a análise de um tema, sendo este processo compreendido como a compensação complementar das deficiências e dos pontos obscuros de cada método isolado. (Flick, 2009, p. 43).

Sendo assim, a metodologia mista desenvolvida pelo grupo ESSA, por fundamentar-se nas proposições de Bernstein, rejeita a objetividade extrema e positivista das metodologias puramente quantitativas, assim como não assume uma postura de forte subjetividade como é preconizado pelas metodologias exclusivamente qualitativas e interpretativas. A relação entre macroestruturas sociais e microestruturas das trocas simbólicas, presente na teoria de Bernstein, pode ser muito bem constatada a partir desses elementos da metodologia.

Como consequência disso, a validação e fiabilidade, segundo Morais e Neves (2007a; 2007b), são elementos caros a essa postura metodológica, haja vista que o foco das pesquisas diz respeito a um campo de fenômenos situados na interface dos universos da instituição educativa e do contexto social dos indivíduos. Isto as impele a produzir respostas que, ao mesmo tempo, estejam conectadas à realidade social e cultural dos indivíduos e que também sejam suficientemente práticas para servirem de fundamento para o estabelecimento de estratégias de ação no âmbito institucional.

Para as autoras, a validade da referida metodologia é garantida, primeiramente, pela teoria rigorosa de alto poder explicativo, como é o caso da teoria de Bernstein. Além disso, a consciência e coerência entre objetivos e coleta de dados, constantes ajustes e reformulações de instrumentos de pesquisa, tempo dilatado de observação, e a existência de interação pessoal e continuada entre investigador e observados, contribuem para garantir essa validade. A fiabilidade, por sua vez, é efetivada mediante a própria triangulação dos dados, como também pela padronização das regras de análise e explicitação de todas as fases da investigação (Morais \& Neves, 2007b).

Por fim, não se pretende olhar para o presente referencial metodológico como um modelo pronto e acabado para os trabalhos de investigação que a partir dele poderão ser conduzidos, mas sim, como um recurso de inspiração teórico-metodológico para que formulemos nossas próprias linguagens de descrição, atentas às realidades que nos suscitam particular interesse. 
A partir desse referencial, pretende-se apresentar a seguir algumas sugestões de categorias de análise potencialmente úteis para a pesquisa no campo do ensino de Geografia, em especial, as que buscam pensar a dimensão curricular da disciplina.

\section{Possíveis categorias de análise para a pesquisa em ensino de Geografia}

De acordo com Bardin (1979) as categorias de análise podem ser entendidas como diferentes dimensões da realidade selecionadas com base em finalidades específicas de estudo, as quais orientam as análises empreendidas em um trabalho de investigação e são um produto da interação entre diferentes bases teóricas e realidades investigadas.

Partindo dessa ideia, e apoiados no referencial bernsteiniano, propõem-se a seguir 5 categorias de análise, as quais poderão sintetizar possíveis problemas de pesquisa e/ou funcionar como orientadoras para a sua construção.

\section{a) Relações inter/intradisciplinares:}

O desenvolvimento do conhecimento e de sua aprendizagem se dá a partir do contato entre diferentes unidades de conhecimento que compõem determinado campo disciplinar específico. No caso do saber geográfico, pode se dizer que essas relações dizem respeito à oposição tanto de diferentes teorias quanto de linguagens, justamente por se considerar sua caracterização como uma disciplina de estrutura discursiva mista, situada na interface entre as ciências naturais e humanas.

Partindo desse pressuposto, acolhe-se aqui a ideia de que a inter/intradisciplinaridade é um elemento central da atividade de desenvolvimento e de apropriação dos discursos verticais. Em outras palavras, essas relações, dentro do modelo teórico-metodológico adotado, são vistas como

(...) relações entre conhecimentos científicos distintos, quer do mesmo nível ou de diferentes níveis de complexidade, e quer dentro da mesma unidade de ensino ou de diferentes unidades de ensino (...), ou mesmo como relações entre o conhecimento declarativo (teoria) e o conhecimento processual (prática) dentro de um determinado conhecimento científico (...). Estas relações podem variar entre muito acentuadas a muito débeis, isto é, com fronteiras muito esbatidas (classificação fraca) ou com fronteiras muito marcadas (classificação forte). (Morais \& Neves, 2012, p. 69).

Essas relações, dessa forma, podem ser vistas a partir da manifestação, em diferentes níveis, da interação entre diferentes unidades de aprendizagem, seja dentro de uma mesma disciplina ou entre disciplinas escolares diferentes.

\section{b) Fronteiras entre saberes conceituais e consensuais:}

Como demonstrado, a teoria de Bernstein (1999) trata os saberes conceituais e consensuais nos termos de discursos verticais e horizontais, respectivamente. Segundo o autor, a fraca delimitação entre essas duas modalidades de discurso pode provocar uma espécie de esvaziamento do currículo escolar, produzindo consequências negativas dentro do processo de promoção da igualdade de acesso ao conhecimento científico na escola.

Ao contrário disso, fronteiras claras entre essas duas modalidades de discurso permitem a construção de currículos que estejam atentos à especificidade contextual e à importância de cada tipo de conhecimento envolvido na construção dos currículos, respeitando sua contextualidade e legitimidade, sem tratá-los como uma "mesma coisa". De acordo com as contribuições de Bernstein e colaboradores, essa é uma condição para que não se negue, justamente aos que mais sofrem com a marginalização social, os saberes construídos social e historicamente pela humanidade.

\section{c) Fronteiras entre discurso instrucional e regulativo:}

O processo de recontextualização, conforme se discutiu anteriormente, está centrado na relação DI/DR, a qual deve ser compreendida como uma relação onde um discurso instrucional sempre estará embutido num discurso regulativo, sendo este último sempre predominante na relação pedagógica. Bernstein afirma que

Os DIs/DRs podem perfeitamente ser fortemente classificados e, portanto, especializados de acordo com os diferentes grupos sociais numa escola e esses DIs/DRs podem perfeitamente ser arranjados numa escala de valor diferencial. Na verdade, pode muito bem ocorrer que certos grupos sejam posicionados apenas no interior do DR, recebendo regulação moral em vez de instrução em competências especializadas. (Bernstein, 1996, p. 279).

Isso quer dizer que em determinados contextos educativos, é possível que o discurso regulativo - composto basicamente por saberes ligados a normas de comportamento, educação moral e conteúdos atitudinais práticos - esteja sendo superexposto ao aluno, em detrimento de uma educação fundamentada em conhecimento conceitual especializado. De acordo com o autor, esse aspecto pode ser um gerador de desigualdades educacionais, privando alunos de determinadas classes sociais ao acesso a determinados tipos de conhecimento. 


\section{d) Critérios de avaliação:}

Consideram-se aqui critérios de avaliação os parâmetros utilizados pelos professores para a legitimação dos textos produzidos pelos alunos em diferentes contextos pedagógicos. De acordo com Bernstein (1996), esses textos podem possuir um teor que se aproxima mais de desempenhos especializados ou estarem mais ligados a competências partilhadas.

No primeiro caso, a atividade pedagógica está fundamentada em um currículo que valoriza de forma mais acentuada o discurso vertical e o conhecimento disciplinar especializado. Já em ambientes pedagógicos onde se privilegia as competências partilhadas, são legítimos e têm maior possibilidade de validação textos que representam saberes adquiridos fora do contexto de aprendizagem escolar, ligados assim ao discurso horizontal.

A validação e legitimação, pelo professor, das diferentes modalidades de textos produzidos pelos estudantes estão em relação direta com o grau de visibilidade que o professor dá aos seus critérios de avaliação. Quanto mais explícitos são os critérios de avaliação maior também será a probabilidade do discurso pedagógico estar concentrado num código de comunicação que privilegia discursos verticais e/ou conceituais.

\section{e) Sequenciamento e compassamento:}

Segundo Bernstein (1996), o compassamento é o ritmo pelo qual a aprendizagem é conduzida, ou seja, a relação da aprendizagem com a variável tempo, enquanto o sequenciamento diz respeito àquilo que vem antes e depois no programa de estudos elaborado pelo professor. De acordo com diferentes contextos educativos, a organização do tempo da aula pode assumir um ritmo mais lento ou mais rápido.

Tais dimensões possuem uma implicação direta na configuração da aula, pois altera a duração dos intervalos destinados às atividades centrais no processo de aprendizagem idealizado pelo professor. De acordo com Galian (2008; 2011), com a finalidade de melhor configurar os espaços e tempos da aula para o aprendizado de crianças em condição de vulnerabilidade, seu compassamento deve respeitar os ritmos que os alunos determinam para si, sem que isso diminua as exigências conceituais no processo. As pesquisas de Galian, fundamentadas no referencial de Bernstein, evidenciam que todos podem aprender, mas em ritmos e tempos que são específicos para cada sujeito.

\section{Considerações finais}

Questões que tradicionalmente frequentam os estudos empreendidos no campo da pesquisa em ensino de Geografia - como a relação entre saberes cotidianos e científicos, a construção de conceitos, a relação entre conhecimentos acadêmicos e escolares, entre a posição social dos sujeitos e seu sucesso e/ou fracasso escolar, e interações entre diferentes temas e/ou conteúdos da própria disciplina (intradisciplinaridade) ou entre diferentes componentes disciplinares (interdisciplinaridade) -, são exemplos de temáticas com as quais a teoria de Bernstein permite diálogo.

As categorias de análise aqui propostas demonstram tais possibilidades e ao mesmo tempo apontam para uma forma específica de encarar os problemas de pesquisa que emergem no campo, indicando uma forma própria de enxergar o futuro da escola. De acordo com o que sugerem algumas pesquisas que se apoiam nessa perspectiva de análise,

(...) uma prática altamente favorável à aprendizagem das crianças de origens sociais diferentes deve mesclar características, tais como: fracas fronteiras entre espaços do professor e dos alunos; relações comunicativas abertas entre professor-alunos e alunos-alunos; critérios de avaliação explícitos; fraco compassamento da aprendizagem; fortes relações intradisciplinares; alto nível de exigência conceitual e alto nível de proficiência investigativa. (Morais et. al., 2004, apud Galian, 2008).

A perspectiva inaugurada por Bernstein e colaboradores, portanto, indica uma concepção autônoma e emancipada de ser humano, sobre a qual se assenta a ideia de que todos, independentemente de sua origem identitária ou de classe social, podem exercer papéis importantes no contexto amplo da vida. A ideia central que orienta o pensamento de Bernstein reside na concepção de que o conhecimento profundo e criativo deve ser oferecido a todos, e que o contexto próximo da vida dos sujeitos não pode ser tomado como um limitador de suas opções. Ao mesmo tempo, como se explicita na citação acima, o respeito aos ritmos de aprendizagem e ao protagonismo do aluno no processo comunicativo também são peças-chave no reconhecimento das culturas que frequentam a escola e na democratização de acesso ao conhecimento poderoso.

Parece-nos, ainda, que desvincular a educação de seu compromisso com o futuro implica um risco muito grande, que é o do reconhecimento que o mundo tal como ele é, já é o bastante. Fechar os olhos e as portas para os marginalizados e para os que sofrem com a opressão do fracasso escolar não parece uma atitude esperada de uma escola que reflete os anseios de uma sociedade que se diz democrática e atenta às desigualdades e diversidades que a compõem. A pesquisa em educação passa a ser vista, a partir daqui, como um agente propositor de mudanças, e por extensão, de um futuro ainda a ser imaginado. 


\section{Referências}

Bardin, L.(1979). Análise de Conteúdo. Lisboa: Edições 70.

Bernstein, B. (1981). Codes, modalities and the process of cultural reproduction: A model. Language and Society, 10, pp. 327-363.

Bernstein, B. (1996). A estruturação do discurso pedagógico: Classes, Código e Controle. Petrópolis: Vozes.

Bernstein, B.(1999). Vertical and horizontal discourse: An essay. British Journal of Sociology of Education, 20,(2), pp. 157-173.

Charlot, B. (2005). Relação com o saber, formação dos professores e globalização. Porto Alegre: Artmed.

Dubet, F. (2008). O que é uma escola justa? A escola das oportunidades. São Paulo: Cortez.

Estudos Sociológicos da Sala de Aula [ESSA]. (2015). O que é: Resumo. Lisboa. Recuperado em 14 de junho, 2016, de http://essa.ie.ulisboa.pt/oquee_resumo_texto.htm.

Flick, U. (2009). Introdução à pesquisa qualitativa. Porto Alegre: Artmed.

Galian, C. V. A. (2008). Contribuições de Bernstein para a descrição e análise das questões ligadas à educação. Revista educativa, 11, n. 2

Galian, C. V. A. (2011). A recontextualização e o nível de exigência conceitual do conhecimento escolar. Educação e Pesquisa, 37, (4).

Libâneo, J. C. (2012). O dualismo perverso da educação pública brasileira: escola do conhecimento para os ricos, escola do acolhimento social para os pobres. Educação e Pesquisa. 38, (1).

Marcuschi, L. A. (1975) Linguagem e classes sociais: introdução crítica à teoria dos códigos linguísticos de Basil Bernstein. Porto Alegre: Editora da URGS.

Morais, A. M, \& Neves, I. P.(2007a). A teoria de Basil Bernstein: alguns aspectos fundamentais. Práxis Educativa. 2, (2).

Morais, A. M, \& Neves, I. P.(2007b). Fazer investigação usando uma abordagem metodológica mista. Revista Portuguesa de Educação. 20, (2).

Morais, A. M, \& Neves, I. P.(2012). Estruturas de conhecimento e exigência conceptual na educação em ciência. Educação, Sociedade \& Culturas, n. 37.

Young, M.(2007). Para que servem as escolas? Educação e Sociedade. 28, (101), pp. 1287-1302.

Young, M. (2011). O futuro da educação em uma sociedade do conhecimento: o argumento radical em defesa de um currículo centrado em disciplinas. Revista Brasileira de Educação, 16, (48), pp. 609-623. 